Mens

revue d'histoire intellectuelle de l'Amérique française

\title{
Jean-Pierre Boyer. Appel à la justice de l'État de Pierre du Calvet. Champion des droits démocratiques au Québec. Québec, Septentrion, 2002.321 p.
}

\section{Nova Doyon}

Volume 4, numéro 1, automne 2003

URI : https://id.erudit.org/iderudit/1024632ar

DOI : https://doi.org/10.7202/1024632ar

Aller au sommaire du numéro

Éditeur(s)

Centre de recherche en civilisation canadienne-française

ISSN

1492-8647 (imprimé)

1927-9299 (numérique)

Découvrir la revue

Citer ce compte rendu

Doyon, N. (2003). Compte rendu de [Jean-Pierre Boyer. Appel à la justice de l'État de Pierre du Calvet. Champion des droits démocratiques au Québec.

Québec, Septentrion, 2002. 321 p.] Mens, 4(1), 111-119.

https://doi.org/10.7202/1024632ar d'utilisation que vous pouvez consulter en ligne.

https://apropos.erudit.org/fr/usagers/politique-dutilisation/ 
manquaient ? Ne faudrait-il pas aborder cette question avec un sens de juste proportion et se départir des illusions de grandeur à propos des capacités de la société québécoise d'alors ?

Il y a un peu plus d'un quart de siècle, Thério écrivait ceci au sujet de l'évêque Bourget: "[II] a canalisé toutes les ressources, toutes les énergies spirituelles et nationales, les a pliées à sa façon de penser et d'agir, leur a donné une forme qui s'est perpétuée à travers le temps et qui nous rejoint... C'est lui qui a indiqué le chemin à tout un peuple » (Ignace Bourget écrivain, 1975, p. 40). Dans son plus récent opuscule, le littérateur, tout en condamnant le parti pris politique, l'absence d'esprit démocratique et la servilité de l'épiscopat, refuse à deux reprises (notes pp. 48, 56) de signaler qu'Ignace Bourget était le seul évêque ayant refusé de rédiger un mandement favorable à la Confédération. Comment expliquer ce silence et, encore plus, ce revirement face à ce qu'il avait écrit précédemment ? Est-il nécessaire aujourd'hui de dépeindre l'évêque tout en noir et ses opposants tout en blanc?

Roberto Perin

Collège Glendon

Université York.

\section{Jean-Pierre Boyer. Appel à la justice de l'État de Pierre du Calvet. Champion des droits démocratiques au Québec. Québec, Septentrion, 2002. 321 p.}

Quiconque s'intéresse à l'histoire des idées au Québec et, plus particulièrement, aux répercussions de l'invasion américaine (1775-1776) et à la circulation des idées démocratiques dans la "Province of Quebec ", trouvera son profit à 
lire le pamphlet de Pierre du Calvet (1735-1786), Appel à la justice de l'État, réédité pour la première fois en version intégrale depuis sa parution à Londres en 1784. Cet Appel... constitue en fait un recueil de lettres dont la publication visait à alerter l'opinion publique pour dénoncer l'arbitraire du régime colonial sous l'administration du gouverneur Frederick Haldimand (1718-1791). Du Calvet, qui avait déjà pris la plume en 1769 pour soumettre au gouverneur Carleton un projet d'uniformisation de l'administration de la justice dans la province, proposait cette fois une réforme constitutionnelle.

Jean-Pierre Boyer, professeur en communications à l'UQAM, féru de la période de la Révolution américaine mais surtout des idées de Thomas Paine, nous offre une édition de l'Appel... enrichie d'une biographie de son auteur ainsi que d'une série de lettres complémentaires éclairant le parcours de du Calvet et, plus largement, l'histoire de la nouvelle colonie britannique à l'heure de la Guerre d'Indépendance américaine (1775-1782). Boyer s'est intéressé à du Calvet dans le dessein avoué de raviver la mémoire de cette « figure de proue du mouvement réformiste canadien du XVIII ${ }^{e}$ siècle et $[\ldots]$ véritable précurseur de la démocratie au Québec » (p. 11).

Huguenot d'origine française, Pierre du Calvet arrive au Canada à la veille de la Cession. Travaillant en Acadie d'abord comme agent de la couronne française puis comme agent du gouvernement britannique, il est ensuite nommé juge de paix par le gouverneur Murray. Marchand prospère de Montréal, du Calvet gagne rapidement les rangs de la bourgeoisie montréalaise. À la suite des événements de 17751776, Londres dépêche le mercenaire suisse Frederick Haldimand pour assurer l'ordre dans la nouvelle colonie britannique. Il assume les fonctions de gouverneur en chef de la province de Québec de juin 1778 à novembre 1784 alors qu'il doit se rendre à Londres pour aller répondre de ses actes de- 
vant le gouvernement britannique. Dès 1779, craignant un soulèvement d'une faction canadienne pro-révolutionaire, Haldimand procède à des arrestations exemplaires. Pierre du Calvet, soupçonné de complicité avec les rebelles américains - il aurait vendu des vivres à l'armée américaine lors de son passage dans la province - , est incarcéré durant plus de trois ans (27 septembre 1780 - 2 mai 1783) sans procès, comme plusieurs autres suspects canadiens.

En regard des autres prisonniers politiques faits par Haldimand, du Calvet a ceci de particulier qu'il décide d'intenter un procès au gouverneur pour violation des droits constitutionnels de sujets du roi de Grande-Bretagne. Aussitôt libéré, il se rend à Londres. Le résultat de ses démarches se faisant attendre, du Calvet décide d'alerter l'opinion publique. Il publie en mars 1784 son mémoire en anglais, The Case of Peter du Calvet, puis, en juillet, son Appel à la justice de l'État. Ce factum est composé, comme il est mentionné en soustitre, de «lettres au Roi, au prince de Galles et aux ministres, avec une lettre à Messieurs les Canadiens [...] une lettre au Général Haldimand lui-même[,] enfin une dernière lettre à Milord Sydney » avec, en plus, une " table raisonnée des matières les plus intéressantes contenues dans ce volume » et une "Introduction ». Dans l'« Épître aux Canadiens », qui constitue la pièce maîtresse du pamphlet, du Calvet vise à conscientiser ses concitoyens. C'est à l'intérieur de cette longue lettre que l'on retrouve ses propositions pour une réforme du système d'administration. Comme le souligne Boyer, en faisant de sa cause, celle d'un homme bafoué dans ses droits et libertés, la cause du peuple canadien, du Calvet se trouve à « enclencher aussi le procès d'un régime despotique d'administration coloniale, au nom d'une collectivité nationale opprimée et réduite à l'esclavage. » (p. 68) Cependant, l'Appel... constitue d'abord le plaidoyer de du Calvet pour dénoncer les 
persécutions que lui ont fait subir les autorités coloniales avant et pendant son emprisonnement. De ce fait, il faut en quelque sorte se méfier du récit autobiographique livré par du Calvet. Pour obtenir l'appui des Canadiens, l'auteur du pamphlet doit présenter son cas comme un exemple, représentatif de ce que d'autres Canadiens pourraient subir. Dans l'« Introduction» de l'Appel..., comme dans l'« Épître aux Canadiens » d'ailleurs, du Calvet se présente en victime d'un tyran, ce qui justifie l'action entreprise contre celui-ci. Ainsi, si le pamphlétaire demande une réparation pour les sévices subis, est-ce aussi pour faire justice à ses compatriotes. De là découlent ses propositions pour une véritable réforme constitutionnelle :

Je commence par l'histoire succincte de mes infortunes, et du succès des voies que la protection des loix m'a ouvertes pour venger avec éclat les violences de mon persécuteur; et je conclurrai pas étaler sous vos yeux les ressources puissantes [de] la Constitution [...], pour briser les chaînes qu'un tyran étranger n'a forgées contre vous $[\ldots]$, et pour vous assurer par vous-mêmes d'un sort national, à l'abri désormais des atteintes de ses semblables : mes efforts ne sont point ici divisés, parce que les intérêts sont d'identité; ma cause est celle de la province de Québec, comme celle de la province de Québec est la mienne... (p. 192)

Pour éclairer la vie de du Calvet, Boyer s'est notamment basé sur les écrits laissés par le personnage (les versions anglaise et française de son pamphlet de même que ses lettres de prison, retranscrites à partir des manuscrits contenus dans le fonds Haldimand de la British Library à Londres). Conscient, comme il le rappelle, du caractère autobiographique de l'œuvre (voir p. 14, note 4), il compare les faits évoqués par du Calvet et les évalue à la lumière d'autres sources primaires ou secondaires traitant de l'époque ou portant sur le person- 
nage. Malgré toutes ces précautions, il apparaît que Boyer ne peut s'empêcher d'« hérö̈ser » son personnage dans la biographie qu'il nous en propose, et ce, pour deux raisons. D'une part, Boyer semble avoir une vision téléologique de l'histoire. Il s'attarde ainsi à montrer les prédispositions de du Calvet qui le conduiront inévitablement à dénoncer l'arbitraire et la tyrannie (elles lui viendraient de son allégeance religieuse). En fait, Boyer s'emploie un peu trop à démontrer la logique du parcours de son personnage, faisant en sorte que la publication de l'Appel... nous apparaisse comme la convergence de toutes ses actions (en accord, bien sûr, avec ses idéaux) voire, dans ce cas-ci, leur aboutissement fatal puisque du Calvet décède à bord du navire qui le ramène de Londres au Canada en 1786.

D'autre part, Boyer semble se laisser gagner par le caractère héroïque du personnage. Bien sûr, en privilégiant la forme autobiographique dans son recueil de lettres, du Calvet doit nécessairement faire son panégyrique pour justifier ses démarches. Si, pour susciter l'adhésion des Canadiens à sa cause, il se présente d'abord comme une victime à laquelle ses compatriotes peuvent s'identifier, il n'hésite pas, par la suite, à faire état de ses qualités, de sa bravoure et de ses exploits passés puisqu'il se propose aussi d'être, en quelque sorte, le libérateur d'un peuple. Boyer, qui a pourtant lu l'analyse du discours de du Calvet que propose Annie Saint-Germain dans son mémoire portant sur L’hérö̈sation dans le discours épistolaire et l'autobiographie : le cas de Pierre du Calvet (1735. 1786) (2000), ne s'en laisse pas moins prendre au jeu.

En effet, il ne peut s'empêcher de croire que son biographé a participé à tous les grands projets idéologiques d'alors (cf. p. 80), allant jusqu'à le considérer, par exemple, comme l'un des fondateurs de l'Académie de Montréal (17781779). Or il est peu probable que cette académie voltairienne 
a bel et bien existé. L'activité présumée de cette société de pensée au sein de la Gazette Littéraire de Montréal, animée par Fleury Mesplet (1734-1794) et Valentin Jautard (1736-1787), devait servir à légitimer la fonction littéraire du journal. Si du Calvet a bel et bien écrit dans la Gazette Littéraire (avril-juin 1779), ses articles n'avaient pourtant rien de littéraire: ils étaient plutôt de nature judiciaire. L'auteur y dénonçait la conduite arbitraire des magistrats de Montréal. Aucune trace manifeste n'atteste de la participation de du Calvet à l'académie montréalaise.

De même, pour expliquer le courroux de du Calvet au sortir de prison et sa détermination à faire le procès de son terrible geôlier, Boyer s'attarde à rappeler les conditions extrêmes de détention du présumé coupable. Il le fait sur la foi du récit qu'en donne du Calvet dans son Appel... mais surtout en se rapportant aux lettres qu'il adressa à Haldimand de prison. Pourtant - et Boyer le rappelle bien - du Calvet ne fut pas le seul prisonnier politique de Haldimand. Le sort des Jautard, Mesplet, Pierre de Sales Laterrière et consorts, dont on peut aussi consulter les lettres de prison dans le fonds Haldimand, n'était guère plus enviable. Aucun d'entre eux n'eut droit à une réponse du gouverneur Haldimand; aucun ne put subir de procès ou même connaitre la cause de son incarcération malgré les demandes répétées qu'ils lui adressèrent depuis leurs cellules. Il reste que, lorsque les prisonniers furent libérés, si certains, défaits, choisirent le silence, d'autres continuèrent à travailler en vue d'obtenir le respect des droits prévus par la constitution britannique dans la colonie. De son côté, du Calvet s'employa à dénoncer les abus du gouvernement colonial et à réclamer, pour les Canadiens, une réforme du système d'administration de la province de Québec. Il est primordial, comme Boyer le fait, de rappeler ses démarches 
qui auront des répercussions importantes sur la vie sociopolitique de la colonie.

Malgré tout, Boyer ne propose pas véritablement une nouvelle lecture ni du pamphlet de du Calvet, ni de la vie et du rôle de l'homme dans la réforme constitutionnelle de la province. N'étant pas historien, il se rapporte surtout à la thèse de Pierre Tousignant consacrée à La genèse et l'avènement de la constitution de 1791 (1971) et favorable au pamphlétaire. De même, il cite abondamment la monographie sur du Calvet produite par Adélard Gascon (1947) dont la thèse, bien qu'elle soit défavorable à du Calvet, est riche de documents éclairants la vie du personnage. On ne sait toutefois pas si Boyer est retourné consulter tous les documents d'archives présentés par l'un ou par l'autre - ce qui est probable pour les pièces qui se trouvent dans le fonds Haldimand - ou s'il s'est fié aux travaux de Gascon et Tousignant. L'opinion des historiens canadiens ou québécois à l'égard de du Calvet a longtemps été polarisée autour des positions représentées par ces deux chercheurs.

S'il est certain que du Calvet fut, comme d'autres Canadiens à l'époque, sinon un sympathisant à la cause des Américains, du moins favorable aux idéaux démocratiques qu'ils proposaient, le rôle qu'il a pu jouer auprès de l'occupant américain dans la province est moins certain. Néanmoins, comme le rappelle Boyer, l'incertitude autour de la contribution de du Calvet à l'invasion a contribué à créer, parmi les historiens, une controverse autour du personnage perçu, par les uns, comme un traître à la nation et, par les autres, comme le précurseur des droits démocratiques au Québec. C'est à Douglas Brymner (et non Brymmer, comme l'écrit Boyer), le premier archiviste canadien, que l'on doit en 1888 une première contre-interprétation du témoignage de du Calvet. Aussi, Boyer, plaidant en faveur de son biographé, s'emploie- 
t-il à démontrer que les preuves de la traitrise de du Calvet sont minces et que la sanction historique portée contre le personnage dépend plutôt de l'allégeance idéologique des historiens qui ont étudié son pamphlet. Comme l'explique Boyer, la controverse est "incontestablement liée aux répercussions de l'Appel à la justice de l'État [...] puisque le livre de du Calvet constitua, pendant plus de cent ans, une référence incontournable pour l'interprétation historique et l'évaluation politique du régime anglais sous l'administration de Haldimand. » (p. 85) De même, comme le note Boyer, l'opinion que les chercheurs se font de du Calvet est nécessairement liée à celle qu'ils ont de Haldimand et crée forcément une seconde polarisation autour de ce dernier personnage : fut-il, comme le prétend du Calvet, un tyran ou un plutôt un gouverneur éclairé, comme ont voulu le démontrer plus tard les historiens conservateurs?

Malgré les controverses autour du personnage de du Calvet, l'ouvrage de Boyer nous montre clairement que ce dernier a véritablement participé au mouvement réformiste canadien. Les propositions contenues dans l'Appel... en vue de réformer le gouvernement colonial ont connu un écho certain dans la province à l'époque. Bien que les marchands britanniques de la province et que certains membres de la bourgeoisie canadienne aient commencé à réclamer une réforme de l'Acte de Québec dès 1783, les citoyens se mobilisèrent véritablement à la suite de la diffusion de l'Appel... au Canada et manifestèrent en grand nombre leur opposition aux termes de l'Acte de 1774. Aussi, si du Calvet ne fut pas le seul à travailler à l'obtention d'une chambre d'assemblée représentative, sans doute fut-il le plus flamboyant des réformistes canadiens : qui d'autre osa alerter l'opinion publique londonienne - et, plus largement, européenne - et dénon- 
cer sans détour le régime politique oppressif de la nouvelle colonie britannique?

La réédition de l'Appel à la justice de l'État de Pierre du Calvet a le mérite de rappeler que les Canadiens ont souhaité et même travaillé à la réforme constitutionnelle de la province de Québec. Là se situe l'essentiel de l'entreprise de J.-P. Boyer.

Nova Doyon

Département d'études littéraires Université du Québec à Montréal

\section{Edmond de Nevers. Lettres de Berlin et d'autres villes d'Europe. Texte établi, présenté et annoté par Hans-Jürgen Lüsebrink. Montréal, Nota bene, 2002. 294 p.}

Né près de Trois-Rivières en 1862 et mort en 1906 à Central Falls, Rhode Island, avant que sa pensée ne puisse pleinement se cristalliser, Edmond Boisvert, devenu Edmond de Nevers en 1884, intimide l'historien des idées. C'est sans doute pour cette raison que si peu de travaux ont été consacrés à la vie et à l'œuvre de ce personnage complexe et énigmatique que l'historien Yvan Lamonde considère comme le premier intellectuel du Canada français. En effet, de Nevers est un homme de lettres sophistiqué et polyglotte - il parle sept ou huit langues, dont le norvégien, et a traduit des œuvres de Matthew Arnold et d'Henrik Ibsen - dont la pensée ne se laisse pas saisir facilement. À première vue, ce libéral à tendance conservatrice, dont les écrits sont à la fois nationalistes et annexionnistes, parait inclassable. 